\title{
Mechanical and physiological effect of partial pressure suit: Experiment and numerical study
}

\author{
Tao Yang ${ }^{\mathrm{a}, \mathrm{b}}$, Li Ding ${ }^{\mathrm{a}}$, Haibo Zhang ${ }^{\mathrm{c}}$, Lihao Xue ${ }^{\mathrm{a}}$, Wei Zhang ${ }^{\mathrm{c}}$, Lianyun Chen ${ }^{\mathrm{c}}$, \\ Huajun Xiao ${ }^{\mathrm{d}}$ and Huiting Qiao ${ }^{\mathrm{a}, *}$ \\ ${ }^{a}$ School of Biological Science and Medical Engineering, Beihang University, Beijing, China \\ ${ }^{\mathrm{b}}$ Inner Mongolia People's Hospital, Hohhot, Inner Mongolia, China \\ ${ }^{\mathrm{c}}$ Aerospace Life-Support Institute Co., Ltd, Xiangyang, Hubei, China \\ ${ }^{\mathrm{d}}$ Institute of Aviation Medicine, Air Force, Beijing, China
}

\begin{abstract}
.
BACKGROUND: During high-altitude flight, the protection of the pilot is vital. A partial pressure suit may affect human physiology, especially circulatory physiology.

OBJECTIVE: The purpose of this study was to investigate how a partial pressure suit works.

METHOD: Ten subjects took part in the flight simulation experiments. Counter pressure at the chest, abdomen, thigh and shank were detected, together with physiological parameters such as heart rate (HR), mean arterial pressure (MAP), stroke volume (SV), cardiac output (CO) and total peripheral resistance (TPR). A numerical model was also established to simulate hemo-physiological effects of the partial pressure suit.

RESULTS: The experiment's results show the non-uniform counter pressure distribution in different parts of the body. There is a linear, proportional relation between TPR and the pressurizing level. HR and MAP increase along with that of the pressure level. SV and CO decrease with the increase of the pressure level. The numerical model simulated the physiological effect of a partial pressure suit. The results were verified by experiment data. The simulation estimated the change of blood flow with the pressure level.

CONCLUSIONS: The numerical model provides a potential way to improve the protection of pilots.
\end{abstract}

Keywords: Partial pressure suit, counter pressure, circulatory physiology, numerical model

\section{Introduction}

With the development of high-performance military aircraft, protection for pilots has become more and more important [1,2]. A partial pressure suit is a type of protection equipment that can provide additional pressure to protect pilots at high altitude and in high-agility flight [3,4]. Because of the significance of a partial pressure suit, some studies have been conducted to evaluate and improve the partial

\footnotetext{
${ }^{*}$ Corresponding author: Huiting Qiao, School of Biological Science and Medical Engineering, Beihang University, Beijing, China. Fax: +86 1082315554; E-mail: qht@ buaa.edu.cn.

0928-7329/17/\$35.00 (c) 2017 - IOS Press and the authors. All rights reserved

This article is published online with Open Access and distributed under the terms of the Creative Commons Attribution NonCommercial License (CC-BY-NC 4.0).
} 
pressure suit [5,6]. Though the physiological effects have been reported in some studies [7,8], a quantitative description is lacking. The relation between additional pressure with the hemodynamic parameters should be established to better understand the mechanism of pressured protection.

When a partial pressure suit is pressurized, a serious biomechanical effect impacts the body, especially the cardiovascular system [9]. Since positive pressure ventilation of oxygen is required under low air pressure, the additional pressure provided by a partial pressure suit acts as the counter pressure to help pilot to breathe. When exposed to 12 kilometer high altitude and $+\mathrm{Gz}$ acceleration, the partial pressure suit will be pressurized. The increasing additional pressure on the abdomen and lower limbs block the blood flow to the lower limbs to avoid hypoxia in the most important tissues [10]. At the same time, the peripheral blood pressure increases, the effective blood circulating in the body is reduced, and cardiac output is decreased [11]. Serious biomechanical effects appear with the body surface pressure caused by the partial pressure suit, while the pressure on the body is not uniform. The partial pressure suit provides a pressure gradient in different parts of the body. For example, the pressure on lower limbs is greater than that on the chest. The non-uniform body surface pressure is strongly related to the effect of the protection from the partial pressure suit, but there are few studies detecting body surface pressure caused by a partial pressure suit and its effect on the cardiovascular system. In this study, the counter pressure on the body surface as well as cardiovascular properties was detected in volunteer experiments.

Although ground-based experiments could be conducted to simulate the cardiovascular response in high altitude and in space [12-14], the quantitative description by a numerical model is preferred to predict the physiological response under special conditions. The numerical modeling method is often employed in cardiovascular system studies [15,16]. A whole body mathematical model has been established to interpret the experimentally-derived intracranial pressure-volume relationship [17]. The lumped parameter model was used by Heldt et al. to describe the cardiovascular response to orthostatic stress [18]. Melek et al. [19] established a second order discrete time model to predict blood pressure dynamics during pitch and roll rotation, and a fuzzy logic model to predict the cardiovascular dynamic changes of head-up/head-down and transitional motions. A lumped circuit model of the pressure sensory baroreflex by Van Heusden et al. [20] studied the viscoelastic stress-relaxation of the systemic veins. Dyachenko et al. [21] developed a model of gravity-dependent effects in the cardiovascular system using numerical integration procedures of a few lumped parameters. Lim et al. developed and optimized the cardiovascular mathematical model to investigate the cardiovascular response of a graded head-up tilt [22]. In spite of these studies on the cardiovascular system, there are few studies on the biomechanical effect of a partial pressure suit. In this study, the lumped whole body model will be developed to explain the protective effect of a partial pressure suit, according to the experimental data.

\section{Methods}

\subsection{Subjects}

The experimental data were collected from 10 males ( $24.6 \pm 4.9$ years old) with a height of $171.6 \pm$ $2.9 \mathrm{~cm}$ and a weight of $66.0 \pm 4.0 \mathrm{~kg}$. All subjects are healthy and have no history of cardiovascular or respiratory disease. Written informed consent was obtained from all participants. The study was approved by the Ethics Committee of Biological Science and Medical Engineering School in Beihang University. 


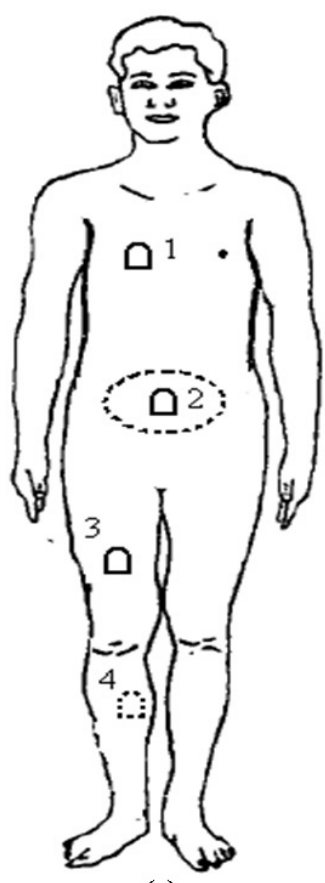

(a)

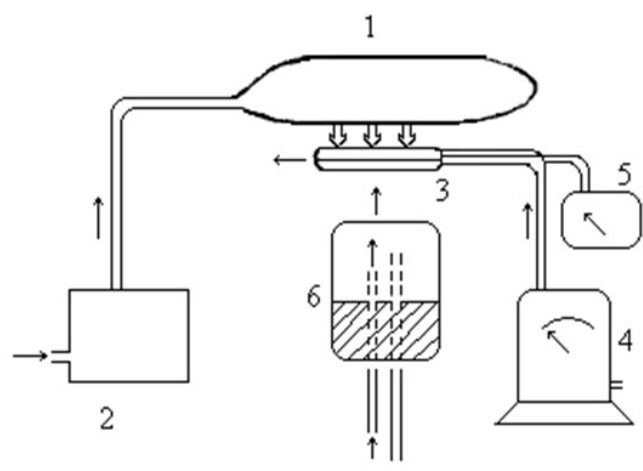

(b)

Fig. 1. Sketch of the measure of counter pressures on body surface: (a) the four different counter pressure measuring points; (b) the schematic diagram of body surface pressure acquisition system (part 1 is the partial pressure suit, part 2 is the pressure control system, part 3 is the pressure bladder, part 4 is the gas flow meter, part 5 is the piezometer, and part 6 is the body part under the pressure bladder).

\subsection{Equipment}

A pressure control system was used together with a partial pressure suit. It has two functions: on the one hand, it provides oxygen through a mask to simulate the positive pressure breathing of the pilot at a high altitude; on the other hand, it can pressurize a partial pressure suit rapidly to the setting value.

The counter pressure on the body surface was measured by a body surface pressure acquisition system. The system was comprised of pressure bladders, which could balance body surface pressure, pressure sensors and an acquisition module, as shown in Fig. 1(b). In this system, skin flap units were placed under the partial pressure suit. The gas meter controlled the gas flow into the pressure bladders until the default pressure was reached. At the same time, the pressure in bladders was detected by the pressure sensors (CGYL-202) and acquisition module.

The physiological parameters were recorded by Portapres ${ }^{\circledR}$ Model 2. Heart rate (HR), mean arterial pressure (MAP), stroke volume (SV), cardiac output (CO) and total peripheral resistance (TPR) were calculated by BeatScope ${ }^{\circledR}$ software.

\subsection{Procedure}

Each subject wore a capstan partial pressure suit with a pressure control system, providing positive pressure oxygen through a mask. Prior to the experiment, all the subjects were trained to master the skills needed for pressurized breathing. 


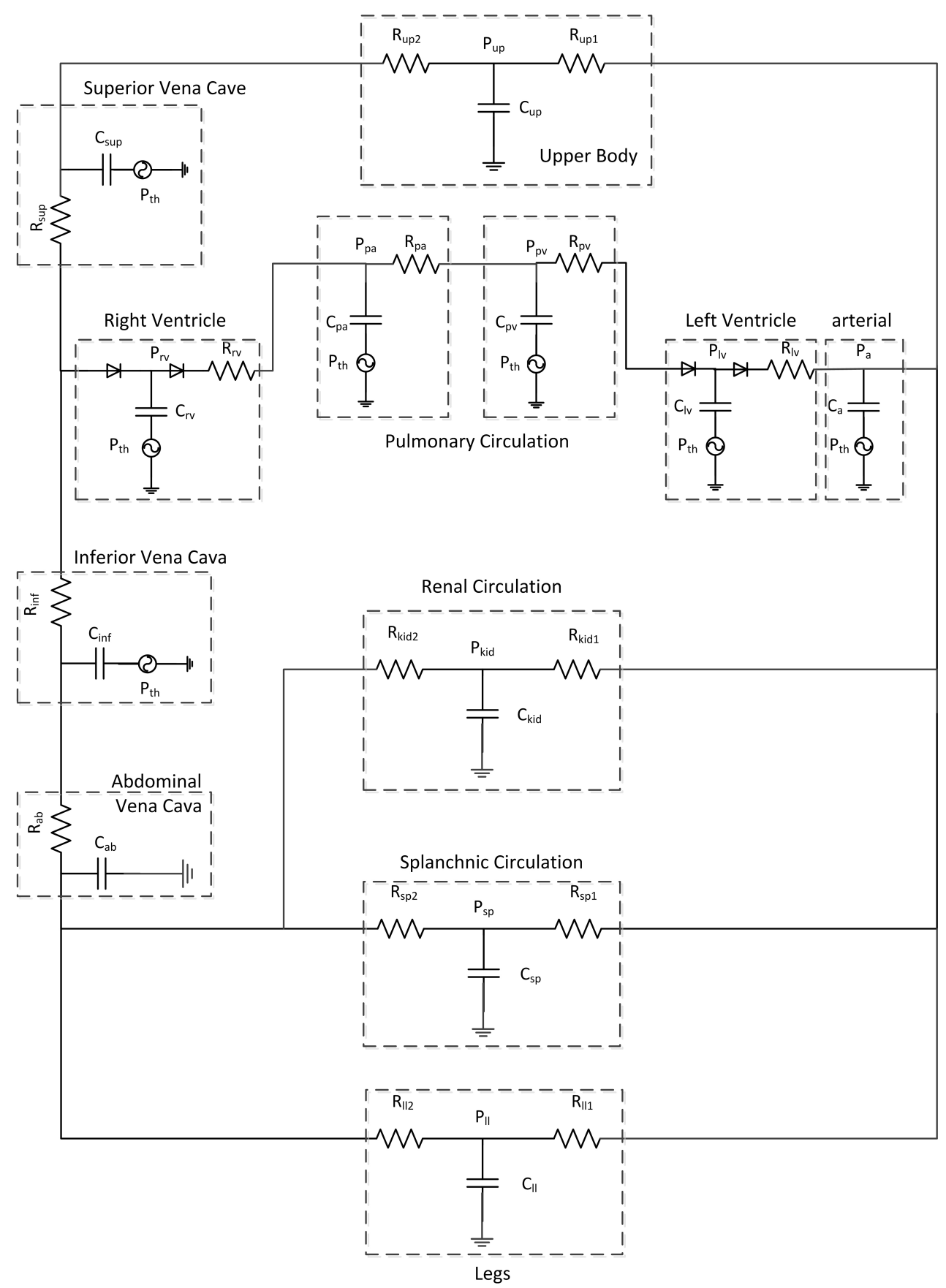

Fig. 2. Model of the cardiovascular system.

In each individual experiment, the partial pressure suit was pressurized at 6 different levels: $0,1.96$, $3.92,5.88,7.84$ and $10.49 \mathrm{kPa}$. At each level, the pressing process lasted $3 \mathrm{~min}$ for each test. There was quite a long time between pressing tests for each subject. When the partial pressure suit was pressurized, the counter pressure on four different parts of the body surface (chest, abdomen, thighs and legs, as 
shown in Fig. 1(a)) were measured by a body pressure acquisition system. At the same time, some physiological parameters, such as heart rate, blood pressure, and cardiac output, were monitored, and the total peripheral resistance was estimated.

Statistical analysis was accomplished using a multivariate software package (SPSS 13.0) with a significance level of $p<0.05$ throughout, and the results were displayed in the form of the mean value \pm the standard deviation

\subsection{Numerical model}

A lumped parameter model was established to describe the hemodynamics of human body [18], as shown in Fig. 2. It included twelve parts: right ventricle, pulmonary veins, pulmonary arteries, left ventricle, aorta, abdominal veins, kidney, splanchnic, lower limbs, upper body, superior vena cava and inferior vena cava. The model was implemented using an electrical analog method. The voltage corresponded to blood pressure, and the current corresponded to the flow rate. $\mathrm{R}$ described the impedance of the some vessel segments, and C stood for the capacitance of the vessel.

The heart was divided into the right ventricle and left ventricle. The ventricles were described by the time-varying elasticity [18]:

$$
E(t)= \begin{cases}E_{\text {dias }}+\frac{E_{s y s}-E_{\text {dias }}}{2} \cdot\left\{1-\cos \left(\pi \cdot \frac{t}{0.3 \sqrt{T}}\right)\right\} & 0 \leqslant t \leqslant 0.3 \sqrt{T} \\ E_{\text {dias }}+\frac{E_{s y s}-E_{\text {dias }}}{2} \cdot\left\{1+\cos \left(2 \pi \cdot \frac{t-0.3 \sqrt{T}}{0.3 \sqrt{T}}\right)\right\} & 0.3 \sqrt{T} \leqslant t \leqslant \frac{3}{2} \cdot 0.3 \sqrt{T} \\ E_{\text {dias }} & \frac{3}{2} \cdot 0.3 \sqrt{T} \leqslant t \leqslant T\end{cases}
$$

where $E_{\text {dias }}$ represents the end-diastolic elastance; $E_{\text {sys }}$ represents the end-systolic elastance; $T$ represents the cardiac cycle.

The model parameters were determined by the ground tests on the pressurized partial pressure suit. The heart rate was estimated by the regression equation with pressure, which was obtained from the experimental data, as with the total peripheral resistance. The linear regression equation was established between pressure and total peripheral resistance. According to the nonuniform counter pressure on body, the vessel resistance of pulmonary veins, pulmonary arteries, splanchnic, lower limbs vessel are set variable. The variable resistance values are linear to the total resistance, which were detected in experiments. The linear scale is related with the counter pressure ratio of chest, abdomen and lower limbs. The other parameters except vessel resistances of pulmonary veins, pulmonary arteries, splanchnic, lower limbs are kept original value of unpressurized situation.

A four-order Runge-Kutta method was used to solve the model differential equations. The results of the simulation were compared with the data of experiments.

\section{Results}

Table 1 shows the counter pressure on the body surface when the capstan partial pressure suit was pressurized from 0 to $10.49 \mathrm{kPa}$. The counter pressure on the body surface increased along with the pressure level, but it was not uniform (as shown in Table 1). The relation between the body surface pressure and the partial pressure suit pressurized level is shown in Fig. 3. The body surface pressures increased rapidly when partial pressure suits were pressurized, and then they increased gradually along with the pressure level. The counter pressure on the thighs was close to that on the shanks. The counter 
Table 1

The counter pressures on the body surface with the partial pressure suit in different level

\begin{tabular}{lcccc}
\hline Pressure level $(\mathrm{kPa})$ & Chest & Abdomen & Thigh & Shank \\
\hline Pre & 0 & 0 & 0 & 0 \\
1.96 & $4.67 \pm 0.66$ & $6.76 \pm 1.21$ & $10.04 \pm 1.42$ & $10.08 \pm 1.95$ \\
3.92 & $5.79 \pm 0.64$ & $8.50 \pm 1.22$ & $11.05 \pm 1.22$ & $11.36 \pm 1.99$ \\
5.88 & $6.18 \pm 0.96$ & $9.57 \pm 1.13$ & $11.18 \pm 1.35$ & $11.82 \pm 2.17$ \\
7.84 & $6.88 \pm 0.67$ & $9.26 \pm 1.86$ & $12.22 \pm 1.41$ & $13.54 \pm 1.05$ \\
10.49 & $7.39 \pm 0.89$ & $10.22 \pm 1.94$ & $13.01 \pm 1.70$ & $13.18 \pm 2.52$ \\
\hline
\end{tabular}

Table 2

The physiological measurements with the partial pressure suit in different pressure level

\begin{tabular}{cccccc}
\hline $\begin{array}{c}\text { Pressure level } \\
(\mathrm{kPa})\end{array}$ & $\begin{array}{c}\mathrm{MAP} \\
(\mathrm{mmHg})\end{array}$ & $\begin{array}{c}\mathrm{TPR} \\
(\mathrm{mmHg} \cdot \mathrm{s} / \mathrm{ml})\end{array}$ & $\begin{array}{c}\mathrm{HR} \\
(\mathrm{times} / \mathrm{min})\end{array}$ & $\begin{array}{c}\mathrm{SV} \\
(\mathrm{ml})\end{array}$ & $\begin{array}{c}\mathrm{CO} \\
(\mathrm{L} / \mathrm{min})\end{array}$ \\
\hline Pre & $96.22 \pm 1.59$ & $1.02 \pm 0.06$ & $79.67 \pm 2.55$ & $70.52 \pm 2.28$ & $5.62 \pm 0.25$ \\
1.96 & $123.73 \pm 1.76$ & $1.71 \pm 0.07$ & $89.68 \pm 3.19$ & $58.46 \pm 3.85$ & $5.24 \pm 0.18$ \\
3.92 & $137.35 \pm 7.23$ & $2.10 \pm 0.14$ & $87.70 \pm 4.18$ & $44.56 \pm 3.85$ & $3.91 \pm 0.19$ \\
5.88 & $149.14 \pm 7.63$ & $2.86 \pm 0.31$ & $91.40 \pm 4.18$ & $41.00 \pm 4.23$ & $3.75 \pm 0.34$ \\
7.84 & $185.09 \pm 4.59$ & $3.71 \pm 0.19$ & $96.51 \pm 2.58$ & $35.66 \pm 1.99$ & $3.44 \pm 0.19$ \\
10.49 & $182.58 \pm 5.64$ & $3.97 \pm 0.40$ & $103.97 \pm 3.72$ & $32.51 \pm 2.90$ & $3.38 \pm 0.30$ \\
\hline
\end{tabular}

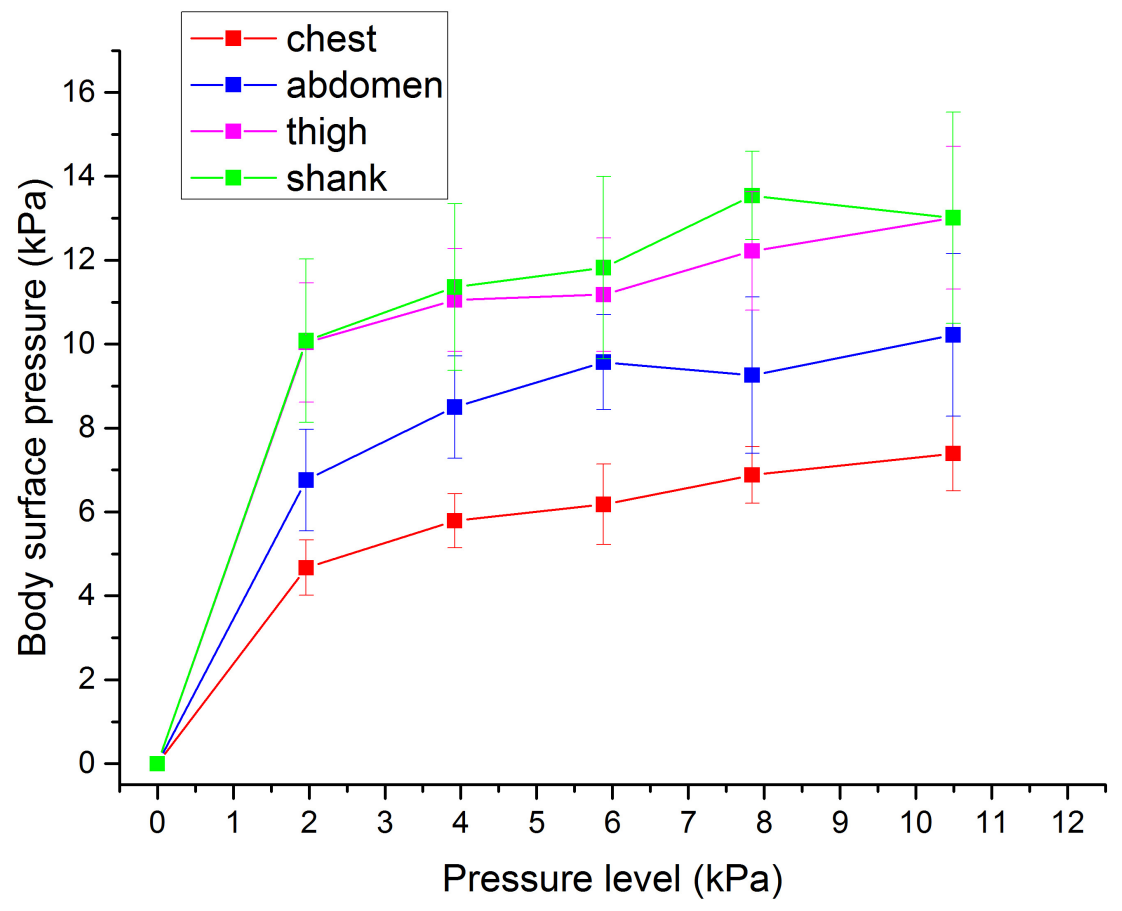

Fig. 3. The counter pressure on the body surface at different pressuring levels.

pressure on low limbs was greater than that on the abdomen. The counter pressure on the chest was the lowest compared with that on the abdomen and lower limbs.

Besides the body surface pressure, some hemodynamic parameters, such as MAP, TPR, HR, SV and $\mathrm{CO}$, were also measured during the pressurized experiments. From the data shown in Table 2, MAP, TPR and HR increased significantly $(p<0.01)$ after pressurization, and SV and CO decreased significantly 


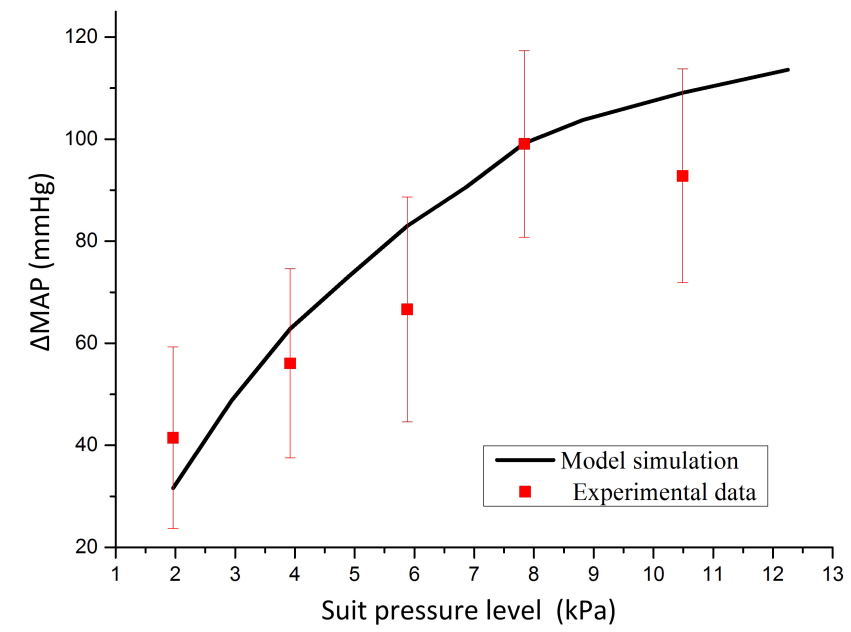

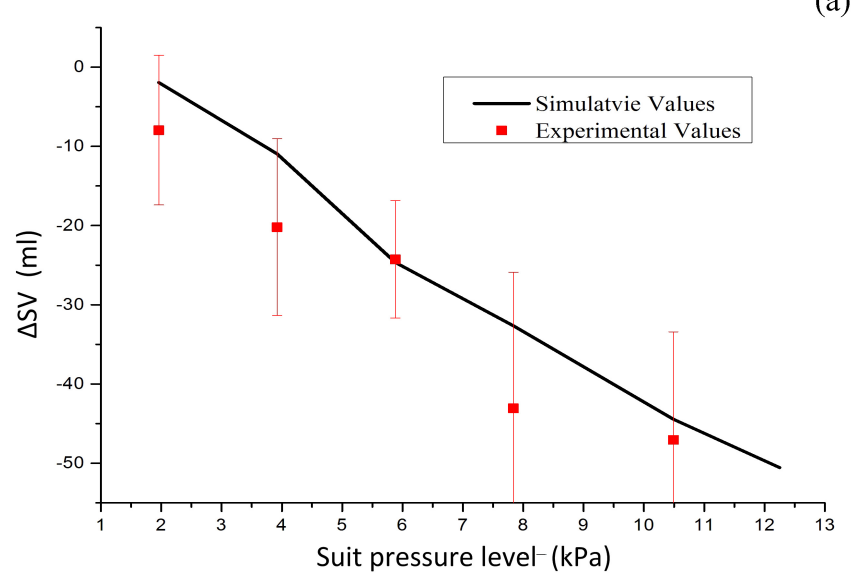

(b)

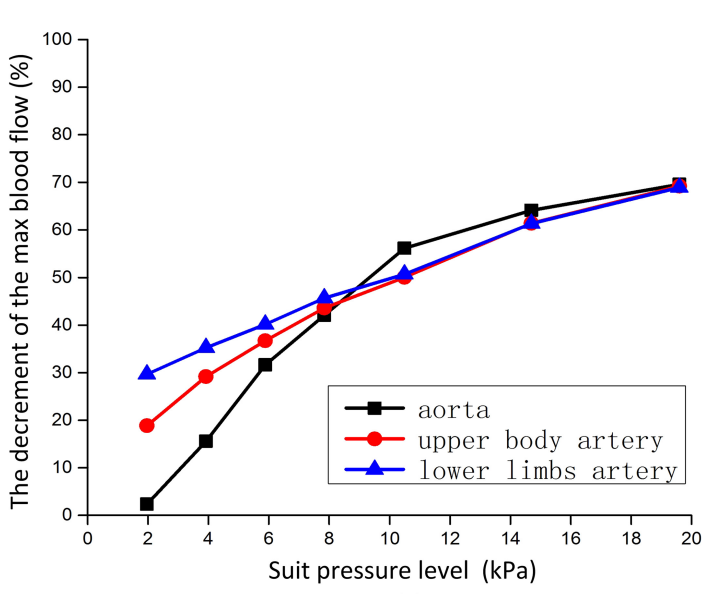

(c)

Fig. 4. The simulation of the model. (a) The simulation of the change in MAP along with the increase of suit pressure level, (b) the simulation of the change in SV along with the increase of suit pressure level, (c) the estimation of the decrement of the max blood flow at different suit pressure levels.

$(p<0.01)$. Both TPR and HR tended to increase gently with the increasing pressure of the partial pressure suit. MAP rose sharply when the partial pressure suit began to be pressurized, and then leveled off gradually along with the increase of pressure. SV and CO both made a sudden drop when pressurized, and then fell gradually along with the increase of pressure.

The results experiments showed that there was a strong linear relationship between the pressure level of pressurized suits and the total peripheral resistance of subjects with partial pressure suits. The linear regression equation is:

$$
\mathrm{TPR}=0.296 * \text { pressure }+1.075
$$

The linear regression coefficient is $\mathrm{R} 2=0.975$. The total peripheral resistance under various pressures was calculated by the regression equation, and used in regulation of the vessel parameters when the partial pressure suit was pressurized. The HR, as an input of the model simulation, was also expressed 
by the linear function of the suit's pressure level:

$$
\mathrm{HR}=2.035 * \text { pressure }+81.28
$$

The linear regression coefficient is $\mathrm{R} 2=0.911$.

By changing the vessel resistance of pulmonary circulation, splanchnic circulation and lower limbs, the model could simulate the change in cardiovascular physiology. The model simulated the MAP and SV, along with the increase of the suit pressure level. The MAP increases with suit pressure levels. The increment of MAP along with suit pressure levels is shown in Fig. 4(a), which is consistent with the experiment data. The SV decreases with suit pressure levels. The decrement of SV along with suit pressure levels is shown in Fig. 4(b), which is consistent with the experiment data.

With the model, the maximum blood flow was estimated at different suit pressure levels, as shown in Fig. 4(c). The decline in maximum blood flow was obvious. When the partial pressure suit was pressurized, the decrease of the maximum blood flow in the lower limbs' arteries is greater than that in the upper body arteries and that in the aorta. But with the increase of the suit pressure level, the decrement of the maximum blood flow in these three parts tends to be the same. The very-high suit pressure level (up to $19.6 \mathrm{kPa}$ ) was also estimated by the model.

\section{Discussion}

The protection provided by the partial pressure suit for pilots during a high altitude flight is of great importance. In this study, the effect of a partial pressure suit on the human body was investigated. The partial pressure suit could exert non-uniform pressure on the surface of the body. At the same time, some of the physiological parameters such as the heart rate, mean arterial pressure, stroke volume and cardiac output, changed. A model was established to describe the hemodynamics of the human body. By varying resistance parameters in the model, the hemodynamic effects of the partial pressure suit were estimated.

When the partial pressure suit was pressurized, non-uniform counter pressure was exerted on the surface of the body. There was a steep change in the counter pressure, when the partial pressure suit began to be pressurized, and then the counter pressure increased linearly with the pressure level. Through measurements taken, it was found that the counter pressure on the thigh and shank was similar, which was a little greater than the counter pressure on the abdomen and chest. Because of the pressure gradient, a partial pressure suit can effectively regulate blood distribution. The higher pressure on lower limbs prevented blood accumulation in the lower body, so that the blood supply to the brain would be sufficient some more important organ blood supply will be sufficient. The non-uniform counter pressure distribution is due to the design of the partial pressure suit. Moreover it affects the hemodynamic physiology to provide protection to pilots.

With a partial pressure suit pressurized, physiological parameters such as the heart rate, peripheral resistance and mean arterial pressure change significantly with the increase in pressure. The peripheral resistance changes linearly with the pressure level of the partial pressure suit. The change in peripheral resistance may be due to the resistance variation in different parts of the model. The variation of peripheral resistance may cause the redistribution of blood flow, and then physiological responses appear. HR and MAP increase along the pressure level, SV and CO decrease with the increase of pressure level.

To describe the effect of a partial pressure suit, a numerical model was established on the basis of the orthostatic stress model by Heldt [18], which was modified in order to apply it to the pressurized partial pressure suit. The influence on circulatory physiology differed with pressurizing levels, due to changes in some of the model parameters, such as the blood flow resistance. Because of the uncertain mechanics 
for HR regulation, the HR in the pressurizing process was used as an input for the model. There are still some limitations of this study. Except HR, other parameters of cardiac function were assumed to be constant when suit pressurized. The effect of partial pressure suit on left ventricle time varying elasticity should be studied further. There is a strong correlation between HR and suit pressure.

The model was validated by the experimental data of MAP and SV. The simulation results are consistent with the experimental data. The model was used to estimate the variation of blood flow in different parts of the body. At the beginning of pressurizing, the percent decrement of maximum blood flow in the lower limbs is greater than that in the upper-body arteries and aorta. The percent decrement of maximum blood flow increased along with the increase of the pressurizing level. The difference between the lower limbs' and upper limbs' percent decrement became less and less, and disappeared when the pressurized pressure was larger than $10 \mathrm{kPa}$. At about the $10 \mathrm{kPa}$ pressurized pressure, the decrease of aorta maximum blood flow became greater than that of the lower limbs. This agrees with the physiological regulation principle. Lower limbs are not as important as the upper part of the body, which includes the brain; thus, the blood distribution in the lower limbs decreased more remarkably to ensure sufficient oxygen supply for the brain. But with the increase in the pressurized pressure, blood in the whole body may change, and the decrease in cardio output may have a more significant effect.

\section{Conclusion}

This study investigated the effects of a partial pressure suit in the process of flight protection by means of an experiment and numerical simulation. The non-uniform counter pressure was recorded with the pressurizing process. MAP, TPR, HR, SV and CO were detected to confirm the physiological effect of a pressurized partial pressure suit. Then a numerical model was established to simulate the hemodynamic changes of the partial pressure suit under different pressure. The model was validated by experimental data and could be used to predict the cardio-physiological effect at different pressurizing levels. This study may provide a way to optimize the design of a partial pressure suit.

\section{Acknowledgments}

This work was supported by National key research and development program (2016YFC0106703, 2015BA102B01, 2016YFC1304305), National Natural Science Foundation of China (51175021), National Key Technology E\&S Program (2014BAK01B05), 111 project (B13003), and the Fundamental Research Funds for the Central Universities of China.

\section{Conflict of interest}

None to report.

\section{References}

[1] Pickard, B.J., Altitude decompression sickness in a pilot wearing a pressure suit above 70,000 feet. Aviation Space and Environmental Medicine, 2003; 74(4): p. 357-359. 
[2] Rudnjanin, S., et al., Loss of consciousness as criterion of + Gz tolerance at Institute of Aviation Medicine MMA during $+G z$ acceleration selective test. Acta Physiol Hung, 2006; 93(4): p. 371-6.

[3] Sears, WJ.. High-altitude pressure protective equipment: a historical perspective. in Raising the Operational Ceiling: a Workshop on the Life Support and Physiological Issues of Flight at 60,000; Feet and Above. 1995: DTIC Document.

[4] Murray, D.H., et al., Pathophysiology, prevention, and treatment of ebullism. Aviation, space, and environmental medicine, 2013; 84(2): p. 89-96.

[5] $\mathrm{Hu}, \mathrm{H}$., et al., The effects of pressurized partial pressure suits on operational ergonomics. International Journal of Industrial Ergonomics, 2015.

[6] Jeon, E.-J., et al., An ergonomic design of flight suit pattern according to wearing characteristics. Proceedings of the Human Factors and Ergonomics Society Annual Meeting, 2011; 55(1): p. 1654-1657.

[7] Qin, Z., et al., Protection and ergonomics analysis about two types of partial pressure suits, in Proceedings of the 13th International Conference on Man-Machine-Environment System Engineering, S. Long and B.S. Dhillon, Editors. 2014; Springer Berlin Heidelberg. p. 401-409.

[8] Eiken, O., et al., textitThe effect of straining maneuvers on G-protection during assisted pressure breathing. Aviation Space and Environmental Medicine, 2003; 74(8): p. 822-826.

[9] Eiken, O., et al., A new hydrostatic anti-G suit vs. a pneumatic anti-G system: Preliminary comparison. Aviation Space and Environmental Medicine, 2002; 73(7): p. 703-708.

[10] Ernsting, J., Present and future compromises in altitude protection in combat aircraft. Operational Medical Issues in Hypo-and Hyperbaric Conditions, 2001; p. 1.

[11] Xiao, H., Physiology of Aviation Oxygen Protective Equipment, 2005; Beijing: Military Medicine Science Press.

[12] Li, X.X., et al., An experimental study on the ergonomics indices of partial pressure suits. Applied Ergonomics, 2013; 44(3): p. 393-403.

[13] Harris, M.A., et al., Differential Branch Pulmonary Artery Regurgitant Fraction Is a Function of Differential Pulmonary Arterial Anatomy and Pulmonary Vascular Resistance. Jacc-Cardiovascular Imaging, 2011; 4(5): p. 506-513.

[14] Schlabs, T., et al., Comparison of cardiovascular and biomechanical parameters of supine lower body negative pressure and upright lower body positive pressure to simulate activity in 1/6 G and 3/8 G. Journal of Applied Physiology, 2013; 115(2): p. 275-284.

[15] Liang, F.Y., et al., Hemodynamic performance of the Fontan circulation compared with a normal biventricular circulation: a computational model study. American Journal of Physiology-Heart and Circulatory Physiology, 2014; 307(7): p. H1056-H1072.

[16] Blanco, PJ.. Feijóo, RA.. A dimensionally-heterogeneous closed-loop model for the cardiovascular system and its applications. Medical Engineering \& Physics, 2013; 35(5): p. 652-667.

[17] Lakin, W.D., et al., A whole-body mathematical model for intracranial pressure dynamics. Journal of Mathematical Biology, 2003; 46(4): p. 347-383.

[18] Heldt, T., et al., Computational modeling of cardiovascular response to orthostatic stress. Journal of applied physiology 2002; 92(3): p. 1239-1254.

[19] Melek, W.W., et al., Modeling of dynamic cardiovascular responses during G-transition-induced orthostatic stress in pitch and roll rotations. IEEE Trans Biomed Eng, 2002; 49(12 Pt 2): p. 1481-90.

[20] van Heusden, K., et al., Mathematical modeling of gravitational effects on the circulation: importance of the time course of venous pooling and blood volume changes in the lungs. Am J Physiol Heart Circ Physiol, 2006; 291(5): p. 21.

[21] Dyachenko, A., et al., Study of numerical integration procedures aimed for computational modeling of gravity-dependent effects in cardiovascular system, in World Congress on Medical Physics and Biomedical Engineering May 26-31, 2012, Beijing, China, M. Long, Editor. 2013; Springer Berlin Heidelberg. p. 2312-2315.

[22] Lim, E., et al., A Cardiovascular Mathematical Model of Graded Head-Up Tilt. Plos One, 2013; 8(10). 